DESY-00-40

\title{
Measurement of Azimuthal Asymmetries in Deep Inelastic Scattering
}

\author{
ZEUS Collaboration
}

\begin{abstract}
The distribution of the azimuthal angle for the charged hadrons has been studied in the hadronic centre-of-mass system for neutral current deep inelastic positron-proton scattering with the ZEUS detector at HERA. Measurements of the dependence of the moments of this distribution on the transverse momenta of the charged hadrons are presented. Asymmetries that can be unambiguously attributed to perturbative QCD processes have been observed for the first time.
\end{abstract}




\section{The ZEUS Collaboration}

J. Breitweg, S. Chekanov, M. Derrick, D. Krakauer, S. Magill, B. Musgrave, A. Pellegrino, J. Repond, R. Stanek, R. Yoshida

Argonne National Laboratory, Argonne, IL, USA ${ }^{p}$

M.C.K. Mattingly

Andrews University, Berrien Springs, MI, USA

G. Abbiendi, F. Anselmo, P. Antonioli, G. Bari, M. Basile, L. Bellagamba, D. Boscherini ${ }^{1}$, A. Bruni, G. Bruni, G. Cara Romeo, G. Castellini ${ }^{2}$, L. Cifarelli ${ }^{3}$, F. Cindolo, A. Contin, N. Coppola, M. Corradi, S. De Pasquale, P. Giusti, G. Iacobucci, G. Laurenti, G. Levi, A. Margotti, T. Massam, R. Nania, F. Palmonari, A. Pesci, A. Polini, G. Sartorelli, Y. Zamora Garcia ${ }^{4}$, A. Zichichi

University and INFN Bologna, Bologna, Italy ${ }^{f}$

C. Amelung, A. Bornheim, I. Brock, K. Coböken, J. Crittenden, R. Deffner, H. Hartmann, K. Heinloth, E. Hilger, P. Irrgang, H.-P. Jakob, A. Kappes, U.F. Katz, R. Kerger, E. Paul, H. Schnurbusch, A. Stifutkin, J. Tandler, K.Ch. Voss, A. Weber, H. Wieber

Physikalisches Institut der Universität Bonn, Bonn, Germany ${ }^{c}$

D.S. Bailey, O. Barret, N.H. Brook ${ }^{5}$, B. Foster ${ }^{6}$, G.P. Heath, H.F. Heath, J.D. McFall, D. Piccioni, E. Rodrigues, J. Scott, R.J. Tapper

H.H. Wills Physics Laboratory, University of Bristol, Bristol, U.K. ${ }^{o}$

M. Capua, A. Mastroberardino, M. Schioppa, G. Susinno

Calabria University, Physics Dept.and INFN, Cosenza, Italy ${ }^{f}$

H.Y. Jeoung, J.Y. Kim, J.H. Lee, I.T. Lim, K.J. Ma, M.Y. Pac ${ }^{7}$

Chonnam National University, Kwangju, Korea ${ }^{h}$

A. Caldwell, W. Liu, X. Liu, B. Mellado, S. Paganis, S. Sampson, W.B. Schmidke, F. Sciulli

Columbia University, Nevis Labs., Irvington on Hudson, N.Y., USA ${ }^{q}$

J. Chwastowski, A. Eskreys, J. Figiel, K. Klimek, K. Olkiewicz, K. Piotrzkowski ${ }^{8}$, M.B. Przybycień, P. Stopa, L. Zawiejski

Inst. of Nuclear Physics, Cracow, Poland ${ }^{j}$

B. Bednarek, K. Jeleń, D. Kisielewska, A.M. Kowal, T. Kowalski, M. Przybycień, E. Rulikowska-Zarȩbska, L. Suszycki, D. Szuba

Faculty of Physics and Nuclear Techniques, Academy of Mining and Metallurgy, Cracow, Poland ${ }^{j}$

A. Kotański

Jagellonian Univ., Dept. of Physics, Cracow, Poland ${ }^{k}$ 
L.A.T. Bauerdick, U. Behrens, J.K. Bienlein, C. Burgard ${ }^{9}$, D. Dannheim, K. Desler, G. Drews, A. Fox-Murphy, U. Fricke, F. Goebel, P. Göttlicher, R. Graciani, T. Haas, W. Hain, G.F. Hartner, D. Hasell ${ }^{10}$, K. Hebbel, K.F. Johnson ${ }^{11}$, M. Kasemann ${ }^{12}$, W. Koch, U. Kötz, H. Kowalski, L. Lindemann ${ }^{13}$, B. Löhr, M. Martínez, M. Milite, T. Monteiro ${ }^{8}$, M. Moritz, D. Notz, F. Pelucchi, M.C. Petrucci, M. Rohde, P.R.B. Saull, A.A. Savin, U. Schneekloth, F. Selonke, M. Sievers, S. Stonjek, E. Tassi, G. Wolf, U. Wollmer,

C. Youngman, W. Zeuner

Deutsches Elektronen-Synchrotron DESY, Hamburg, Germany

C. Coldewey, A. Lopez-Duran Viani, A. Meyer, S. Schlenstedt, P.B. Straub

DESY Zeuthen, Zeuthen, Germany

G. Barbagli, E. Gallo, P. Pelfer

University and INFN, Florence, Italy ${ }^{f}$

G. Maccarrone, L. Votano

INFN, Laboratori Nazionali di Frascati, Frascati, Italy ${ }^{f}$

A. Bamberger, A. Benen, S. Eisenhardt ${ }^{14}$, P. Markun, H. Raach, S. Wölfle

Fakultät für Physik der Universität Freiburg i.Br., Freiburg i.Br., Germany ${ }^{c}$

P.J. Bussey, A.T. Doyle, S.W. Lee, N. Macdonald, G.J. McCance, D.H. Saxon, L.E. Sinclair,

I.O. Skillicorn, R. Waugh

Dept. of Physics and Astronomy, University of Glasgow, Glasgow, U.K. ${ }^{\circ}$

I. Bohnet, N. Gendner, U. Holm, A. Meyer-Larsen, H. Salehi, K. Wick

Hamburg University, I. Institute of Exp. Physics, Hamburg, Germany ${ }^{c}$

A. Garfagnini, I. Gialas ${ }^{15}$, L.K. Gladilin ${ }^{16}$, D. Kçira ${ }^{17}$, R. Klanner, E. Lohrmann, G. Poelz, F. Zetsche

Hamburg University, II. Institute of Exp. Physics, Hamburg, Germany ${ }^{c}$

R. Goncalo, K.R. Long, D.B. Miller, A.D. Tapper, R. Walker

Imperial College London, High Energy Nuclear Physics Group, London, U.K. ${ }^{\circ}$

U. Mallik

University of Iowa, Physics and Astronomy Dept., Iowa City, USA ${ }^{p}$

P. Cloth, D. Filges

Forschungszentrum Jülich, Institut für Kernphysik, Jülich, Germany

T. Ishii, M. Kuze, K. Nagano, K. Tokushuku ${ }^{18}$, S. Yamada, Y. Yamazaki

Institute of Particle and Nuclear Studies, KEK, Tsukuba, Japan ${ }^{g}$

S.H. Ahn, S.B. Lee, S.K. Park

Korea University, Seoul, Korea ${ }^{h}$

H. Lim, I.H. Park, D. Son

Kyungpook National University, Taegu, Korea ${ }^{h}$

F. Barreiro, G. García, C. Glasman ${ }^{19}$, O. Gonzalez, L. Labarga, J. del Peso, I. Redondo ${ }^{20}$, J. Terrón

Univer. Autónoma Madrid, Depto de Física Teórica, Madrid, Spain ${ }^{n}$

M. Barbi, F. Corriveau, D.S. Hanna, A. Ochs, S. Padhi, M. Riveline, D.G. Stairs, M. Wing

McGill University, Dept. of Physics, Montréal, Québec, Canada ${ }^{a},{ }^{b}$ 
T. Tsurugai

Meiji Gakuin University, Faculty of General Education, Yokohama, Japan

V. Bashkirov ${ }^{21}$, B.A. Dolgoshein

Moscow Engineering Physics Institute, Moscow, Russia ${ }^{l}$

R.K. Dementiev, P.F. Ermolov, Yu.A. Golubkov, I.I. Katkov, L.A. Khein, N.A. Korotkova, I.A. Korzhavina, V.A. Kuzmin, O.Yu. Lukina, A.S. Proskuryakov, L.M. Shcheglova, A.N. Solomin,

N.N. Vlasov, S.A. Zotkin

Moscow State University, Institute of Nuclear Physics, Moscow, Russia ${ }^{m}$

C. Bokel, M. Botje, N. Brümmer, J. Engelen, S. Grijpink, E. Koffeman, P. Kooijman, S. Schagen, A. van Sighem, H. Tiecke, N. Tuning, J.J. Velthuis, J. Vossebeld, L. Wiggers, E. de Wolf

NIKHEF and University of Amsterdam, Amsterdam, Netherlands ${ }^{i}$

D. Acosta ${ }^{22}$, B. Bylsma, L.S. Durkin, J. Gilmore, C.M. Ginsburg, C.L. Kim, T.Y. Ling

Ohio State University, Physics Department, Columbus, Ohio, USA ${ }^{p}$

S. Boogert, A.M. Cooper-Sarkar, R.C.E. Devenish, J. Große-Knetter ${ }^{23}$, T. Matsushita, O. Ruske,

M.R. Sutton, R. Walczak

Department of Physics, University of Oxford, Oxford U.K. ${ }^{\circ}$

A. Bertolin, R. Brugnera, R. Carlin, F. Dal Corso, U. Dosselli, S. Dusini, S. Limentani, M. Morandin, M. Posocco, L. Stanco, R. Stroili, C. Voci

Dipartimento di Fisica dell' Università and INFN, Padova, Italy ${ }^{f}$

L. Adamczyk ${ }^{24}$, L. Iannotti ${ }^{24}$, B.Y. Oh, J.R. Okrasiński, W.S. Toothacker, J.J. Whitmore

Pennsylvania State University, Dept. of Physics, University Park, PA, USA ${ }^{q}$

Y. Iga

Polytechnic University, Sagamihara, Japan ${ }^{g}$

G. D'Agostini, G. Marini, A. Nigro

Dipartimento di Fisica, Univ. 'La Sapienza' and INFN, Rome, Italy ${ }^{f}$

C. Cormack, J.C. Hart, N.A. McCubbin, T.P. Shah

Rutherford Appleton Laboratory, Chilton, Didcot, Oxon, U.K. ${ }^{\circ}$

D. Epperson, C. Heusch, H.F.-W. Sadrozinski, A. Seiden, R. Wichmann, D.C. Williams

University of California, Santa Cruz, CA, USA ${ }^{p}$

N. Pavel

Fachbereich Physik der Universität-Gesamthochschule Siegen, Germany ${ }^{c}$

H. Abramowicz ${ }^{25}$, S. Dagan ${ }^{26}$, S. Kananov ${ }^{26}$, A. Kreisel, A. Levy ${ }^{26}$

Raymond and Beverly Sackler Faculty of Exact Sciences, School of Physics, Tel-

Aviv University,

Tel-Aviv, Israel ${ }^{e}$

T. Abe, T. Fusayasu, K. Umemori, T. Yamashita

Department of Physics, University of Tokyo, Tokyo, Japan ${ }^{g}$

R. Hamatsu, T. Hirose, M. Inuzuka, S. Kitamura ${ }^{27}$, T. Nishimura

Tokyo Metropolitan University, Dept. of Physics, Tokyo, Japan ${ }^{g}$ 
M. Arneodo ${ }^{28}$, N. Cartiglia, R. Cirio, M. Costa, M.I. Ferrero, S. Maselli, V. Monaco, C. Peroni, M. Ruspa, R. Sacchi, A. Solano, A. Staiano

Università di Torino, Dipartimento di Fisica Sperimentale and INFN, Torino, Italy ${ }^{f}$

M. Dardo

II Faculty of Sciences, Torino University and INFN - Alessandria, Italy ${ }^{f}$

D.C. Bailey, C.-P. Fagerstroem, R. Galea, T. Koop, G.M. Levman, J.F. Martin, R.S. Orr, S. Polenz, A. Sabetfakhri, D. Simmons

University of Toronto, Dept. of Physics, Toronto, Ont., Canada ${ }^{a}$

J.M. Butterworth, C.D. Catterall, M.E. Hayes, E.A. Heaphy, T.W. Jones, J.B. Lane, B.J. West

University College London, Physics and Astronomy Dept., London, U.K. ${ }^{o}$

J. Ciborowski, R. Ciesielski, G. Grzelak, R.J. Nowak, J.M. Pawlak, R. Pawlak, B. Smalska,

T. Tymieniecka, A.K. Wróblewski, J.A. Zakrzewski, A.F. Żarnecki

Warsaw University, Institute of Experimental Physics, Warsaw, Poland ${ }^{j}$

M. Adamus, T. Gadaj

Institute for Nuclear Studies, Warsaw, Poland ${ }^{j}$

O. Deppe, Y. Eisenberg, D. Hochman, U. Karshon ${ }^{26}$

Weizmann Institute, Department of Particle Physics, Rehovot, Israel ${ }^{d}$

W.F. Badgett, D. Chapin, R. Cross, C. Foudas, S. Mattingly, D.D. Reeder, W.H. Smith, A. Vaiciulis ${ }^{29}$, T. Wildschek, M. Wodarczyk

University of Wisconsin, Dept. of Physics, Madison, WI, USA ${ }^{p}$

A. Deshpande, S. Dhawan, V.W. Hughes

Yale University, Department of Physics, New Haven, CT, USA ${ }^{p}$

S. Bhadra, C. Catterall, J.E. Cole, W.R. Frisken, R. Hall-Wilton, M. Khakzad, S. Menary

York University, Dept. of Physics, Toronto, Ont., Canada ${ }^{a}$ 
${ }^{1}$ now visiting scientist at DESY

2 also at IROE Florence, Italy

3 now at Univ. of Salerno and INFN Napoli, Italy

${ }^{4}$ supported by Worldlab, Lausanne, Switzerland

${ }^{5}$ PPARC Advanced fellow

${ }^{6}$ also at University of Hamburg, Alexander von Humboldt Research Award

7 now at Dongshin University, Naju, Korea

8 now at CERN

${ }^{9}$ now at Barclays Capital PLC, London

10 now at Massachusetts Institute of Technology, Cambridge, MA, USA

11 visitor from Florida State University

12 now at Fermilab, Batavia, IL, USA

13 now at SAP A.G., Walldorf, Germany

14 now at University of Edinburgh, Edinburgh, U.K.

15 visitor of Univ. of Crete, Greece, partially supported by DAAD, Bonn - Kz. A/98/16764

16 on leave from MSU, supported by the GIF, contract I-0444-176.07/95

17 supported by DAAD, Bonn - Kz. A/98/12712

18 also at University of Tokyo

19 supported by an EC fellowship number ERBFMBICT 972523

20 supported by the Comunidad Autonoma de Madrid

${ }^{21}$ now at Loma Linda University, Loma Linda, CA, USA

22 now at University of Florida, Gainesville, FL, USA

23 supported by the Feodor Lynen Program of the Alexander von Humboldt foundation

${ }^{24}$ partly supported by Tel Aviv University

25 an Alexander von Humboldt Fellow at University of Hamburg

${ }^{26}$ supported by a MINERVA Fellowship

27 present address: Tokyo Metropolitan University of Health Sciences, Tokyo 116-8551, Japan

28 now also at Università del Piemonte Orientale, I-28100 Novara, Italy

29 now at University of Rochester, Rochester, NY, USA 
a supported by the Natural Sciences and Engineering Research Council of Canada (NSERC)

${ }^{b}$ supported by the FCAR of Québec, Canada

$c$ supported by the German Federal Ministry for Education and Science, Research and Technology (BMBF), under contract numbers 057BN19P, 057FR19P, 057HH19P, 057HH29P, 057SI75I

$d$ supported by the MINERVA Gesellschaft für Forschung GmbH, the German Israeli Foundation, the Israel Science Foundation, the Israel Ministry of Science and the Benozyio Center for High Energy Physics

$e$ supported by the German-Israeli Foundation, the Israel Science Foundation, the U.S.-Israel Binational Science Foundation, and by the Israel Ministry of Science

$f \quad$ supported by the Italian National Institute for Nuclear Physics (INFN)

$g \quad$ supported by the Japanese Ministry of Education, Science and Culture (the Monbusho) and its grants for Scientific Research

$h$ supported by the Korean Ministry of Education and Korea Science and Engineering Foundation

${ }^{i} \quad$ supported by the Netherlands Foundation for Research on Matter (FOM)

$j$ supported by the Polish State Committee for Scientific Research, grant No. 112/E356/SPUB/DESY/P03/DZ 3/99, 620/E-77/SPUB/DESY/P-03/ DZ 1/99, 2P03B03216, 2P03B04616, 2P03B03517, and by the German Federal Ministry of Education and Science, Research and Technology (BMBF)

$k$ supported by the Polish State Committee for Scientific Research (grant No. 2P03B08614 and 2P03B06116)

$l$ partially supported by the German Federal Ministry for Education and Science, Research and Technology (BMBF)

$m$ supported by the Fund for Fundamental Research of Russian Ministry for Science and Education and by the German Federal Ministry for Education and Science, Research and Technology (BMBF)

$n$ supported by the Spanish Ministry of Education and Science through funds provided by CICYT

o supported by the Particle Physics and Astronomy Research Council

$p$ supported by the US Department of Energy

$q \quad$ supported by the US National Science Foundation 


\section{Introduction}

Semi-inclusive processes in deep inelastic scattering (DIS) are of importance because they can be used to test the perturbative Quantum Chromodynamic (QCD) description of hadron production via parton fragmentation. An observable of particular interest is the distribution of the azimuthal angle, $\phi$, (measured in the hadronic centre-of-mass frame, HCM) between the lepton scattering plane, defined by the incoming and outgoing lepton momenta, and the hadron production plane, defined by the exchanged virtual boson and an outgoing hadron (Fig. 1).

Asymmetries in the $\phi$ distribution, i.e. terms proportional to $\cos \phi$ and $\cos 2 \phi$ arise whenever a non-zero transverse momentum, in the HCM frame, is present in the scattering process. Consequently both non-perturbative and perturbative QCD effects [1] [7] give rise to these asymmetries. The azimuthal dependence of parton production has the form

$$
d \sigma / d \phi=A+B \cos \phi+C \cos 2 \phi .
$$

This form results from the polarisation of the exchanged virtual boson. The coefficients $B$ and $C$ depend on the helicities of the final-state parton(s), partonic transverse momenta and on colour coherence [5]. The $\cos 2 \phi$ term is expected from interference of amplitudes arising from the +1 and -1 helicity components of the transversely-polarised part of the exchanged boson, whereas transverse/longitudinal interference gives rise to the $\cos \phi$ term.

The asymmetry that results from the intrinsic momentum of a quark in the proton is referred to as the non-perturbative asymmetry. Since the intrinsic momentum is small, this asymmetry should fall rapidly with increasing transverse momentum of the measured hadron and with increasing $Q^{2}$ [2], where $Q^{2} \equiv-q^{2}$ is the negative square of the four-momentum of the virtual exchanged boson. In particular, the term $C$ is small at high $Q^{2}$ for the non-perturbative effect.

In contrast, the asymmetry associated with leading-order terms in perturbative QCD calculations, the perturbative asymmetry, is weakly dependent on $Q^{2}$ and persists at high transverse momenta. The perturbative QCD contribution to terms $B$ and $C$ is large at leading order (LO) in $\alpha_{s}$. At this order, two processes contribute to DIS: QCD-Compton scattering (QCDC, $\gamma^{*} q \rightarrow q g$ ) and boson-gluon fusion (BGF, $\gamma^{*} g \rightarrow q \bar{q}$ ). For the QCDC process the scattered quark preferentially appears at $\phi$ near $180^{\circ}$ whilst for the BGF process the $\phi$ dependence has a symmetry about $180^{\circ}$, as a consequence of the quark/antiquark final state. The BGF process is the dominant contribution to the $\cos 2 \phi$ term, while the QCDC contribution dominates the $\cos \phi$ term. The $\cos 2 \phi$ term can be unambiguously attributed to perturbative QCD processes in the high $Q^{2}$ region under investigation.

Terms in $\sin \phi$ and $\sin 2 \phi$ are also theoretically expected only for deep inelastic scattering with polarised leptons or charged current reactions. Even in these cases, their magnitude is however estimated to be substantially smaller than terms in $\cos \phi$ and $\cos 2 \phi[7]$.

The form of Eq. 1 is expected to be maintained for single particle production [5, 7], since high-momentum hadrons are produced close to the direction of the parton. Measurable perturbative and non-perturbative predictions for the asymmetries in hadron production require the use of an appropriate fragmentation function or hadronisation mechanism. Note that in order to observe the 
$\cos \phi$ asymmetry, a selection procedure is required which consistently associates leading hadrons produced from either quarks or gluons [5,77. This is described in Section 2. The transverse momenta arising from the fragmentation itself does not contribute to the asymmetry but only smears the observed distribution [5].

The event kinematics of DIS are determined by $Q^{2}$, and one of the two Bjorken scaling variables $x=Q^{2} / 2 P \cdot q$ or $y=Q^{2} / x s$, where $P$ is the four-momentum of the incoming proton and $\sqrt{s}$ is the positron-proton centre-of-mass energy. The kinematic region studied is $0.2<y<0.8$ and $0.01<x<0.1$, corresponding to a $Q^{2}$ range $180<Q^{2}<7220 \mathrm{GeV}^{2}$.

Charged particles are selected over a defined range of $p_{T}$ values, where $p_{T}$ is the transverse momentum with respect to the $\gamma^{*} P$ axis in the HCM system. Results for the asymmetry are presented as a function of $p_{c}$, the minimum value of $p_{T}$ of the selected charged particles, since the QCD predictions depend on this variable. The measurements are compared to theoretical expectations.

Previous studies of single hadron production in neutral current DIS have observed a $\cos \phi$ term that was attributed to non-perturbative effects [8, 9]. Here, for the first time, the observation of both $\cos \phi$ and $\cos 2 \phi$ terms are reported. As discussed above, the $\cos 2 \phi$ term can be unambiguously attributed to perturbative QCD processes.

\section{Experimental Setup and Event Selection}

The data presented here were taken at the positron-proton collider HERA using the ZEUS detector. The $38 \mathrm{pb}^{-1}$ of data were taken in 1996 and 1997, when HERA operated with positrons of energy $E_{e}=27.5 \mathrm{GeV}$ and protons with energy $820 \mathrm{GeV}$. A detailed description of the ZEUS detector can be found elsewhere [10, 11].

Throughout this paper the standard ZEUS right-handed coordinate system is used, in which $X=Y=Z=0$ is the nominal interaction point, the positive $Z$-axis points in the direction of the proton beam (referred to as the forward direction) and the $X$-axis is horizontal, pointing towards the centre of HERA. The polar angle, $\theta$, is defined with respect to the positive $Z$ direction.

The key component for this analysis is the central tracking detector (CTD) 12 which operates in a magnetic field of $1.43 \mathrm{~T}$ provided by a thin superconducting solenoid. The CTD is a drift chamber consisting of 72 cylindrical layers, covering the region $15^{\circ}<\theta<164^{\circ}$. The transverse momentum resolution for fulllength tracks is $\sigma\left(p_{T}\right) / p_{T}=0.0058 p_{T} \oplus 0.0065 \oplus 0.0014 / p_{T}\left(p_{T}\right.$ in $\left.\mathrm{GeV}\right)$. The interaction vertex is measured using the CTD with a typical resolution along (transverse to) the beam direction of $0.4(0.1) \mathrm{cm}$.

Also important for this analysis is the uranium-scintillator sampling calorimeter (CAL) [13], which surrounds the solenoid. The CAL consists of 5918 cells, organised in three sections, the forward (FCAL), barrel (BCAL) and rear (RCAL), with longitudinal segmentation into electromagnetic and hadronic sections. The energy resolutions, as measured in test beams, are $\sigma / E=0.18 / \sqrt{E(\mathrm{GeV})}$ and $0.35 / \sqrt{E(\mathrm{GeV})}$ for electrons and hadrons, respectively.

The angular coverage of the ZEUS detector allows the kinematic variables $x$ and $y$ to be reconstructed in a variety of ways using combinations of energies and angles of the positron and the hadronic system. Variables calculated only from the measurements of the energy, $E_{e}^{\prime}$, and angle, $\theta_{e}$, of the scattered positron are denoted with the subscript $e$, whilst those calculated from the hadronic 
system measurements, using the Jacquet-Blondel method [14], are denoted by the subscript $J B$. Variables calculated by these approaches are used only in the event selection. In the double angle method [15], denoted by $D A$, the kinematic variables are determined using $\theta_{e}$ and the angle $\gamma_{H}$ (which is the direction of the struck quark in the QPM), evaluated using the CAL cells corresponding to the hadronic final state.

The $D A$ method was used throughout this analysis for the calculation of the boosts and the kinematic variables because it is less sensitive than other methods to systematic uncertainties in the CAL energy measurement.

Corrections are applied [16] for hadronic energy loss in inactive material in front of the calorimeter and for the backscattering of particles into the BCAL or RCAL from hadron jets in the FCAL direction.

The triggering and online event selections were identical to those used for the ZEUS measurement of the structure function $F_{2}$ [17].

Further selection criteria were applied both to ensure accurate reconstruction of the kinematic variables and to increase the purity of the sample by eliminating background from photoproduction processes:

- $E_{e}^{\prime} \geq 10 \mathrm{GeV}$, to achieve a high purity sample of DIS events;

- $y_{e} \leq 0.95$, to reduce the photoproduction background;

- $y_{J B} \geq 0.04$, to give sufficient accuracy for $D A$ reconstruction of $Q^{2}$ and $x$

- $40 \leq \sum\left(E-p_{Z}\right) \leq 60 \mathrm{GeV}$, where the summation is over all calorimeter cells, to remove photoproduction events and events with large radiative corrections;

- $|X|>16 \mathrm{~cm}$ or $|Y|>16 \mathrm{~cm}$, where $X$ and $Y$ are the impact position of the positron on the RCAL, to avoid the region adjacent to the rear beampipe;

- $-40<Z_{\text {vertex }}<50 \mathrm{~cm}$, to reduce background events from processes other than ep collisions;

- $0.01<x_{D A}<0.1$ and $0.2<y_{D A}<0.8$, to ensure $\gamma_{H}$ lies within the CTD acceptance.

The reconstructed tracks used in the charged particle analysis are associated with the primary event vertex and are required to have $p_{T}^{\text {lab }}>150 \mathrm{MeV}$ and $\left|\eta^{\text {lab }}\right|<1.75$, where $\eta^{\text {lab }}$ is the pseudorapidity, given by $-\ln (\tan (\theta / 2))$. These cuts select the region of CTD acceptance where the detector response and systematics are best understood.

Since the gluon fragmentation function is 'softer' than that of the quarks, the hadron-quark correlation can be enhanced by selecting 'leading' charged particles. This was accomplished by cutting on the Lorentz-invariant variable $z_{h}=P \cdot p_{h} / P \cdot q$, where $p_{h}$ is the track or particle four-vector. The tracks were selected if $0.2<z_{h}<1.0$; no selection biases arise from the $\eta$ cut applied in the laboratory frame.

The number of events that pass the event selection is just over 13,800; approximately 7,700 charged tracks satisfy the $z_{h}$ criteria. In principle, more than one entry per event is permitted. However, as can be seen from the above numbers, such an occurrence is rare. 


\section{QCD models and event simulation}

Monte Carlo event simulation is used to correct for acceptance and resolution effects. The detector simulation is based on the GEANT 3.13 program [18].

To calculate correction factors, neutral current DIS events were generated via DJANGO6 2.4 [19], using HERACLES 4.5.2 [20], which incorporates first-order electroweak corrections. The parton cascade was modelled with the colour dipole model (CDM), using the ARIADNE 4.10 21] program, in which colour coherence effects are implicitly included in the formalism of the parton cascade. The Lund string fragmentation model 22 is used for the hadronisation, as implemented in JETSET 7.4 [23]. For the analysis of the data, a Monte Carlo sample of $120 \mathrm{k}$ events, corresponding to an integrated luminosity of $67 \mathrm{pb}^{-1}$, was generated with $0.13<y<1.0$ and $0.007<x<0.3$ and the CTEQ4D [24] parameterisation of the proton's parton distribution functions. The CTEQ4D parame-

terisation has been shown to describe satisfactorily the HERA measurements of the proton structure function $F_{2}$ [25, 26] in the kinematic regions investigated.

For systematic checks, an additional sample of $120 \mathrm{k}$ events was generated using the DJANGO6 and LEPTO 6.5 [27] Monte Carlo programs. The parton shower option, matched to the $\mathcal{O}\left(\alpha_{s}\right)$ matrix elements (ME+PS), was used to simulate the parton cascade. The coherence effects in the final-state cascade are included by angular ordering of successive parton emissions. For the hadronisation the Lund string model was used and for the parameterisation of the parton distribution functions CTEQ4D was used. The LEPTO sample was generated with the soft colour interactions 29 turned off.

The ARIADNE sample was generated with the high $Q^{2}$ modification developed during the 1998 HERA Monte Carlo workshop [28]. A version of ARIADNE 4.10, with a corrected implementation of the $\phi$ dependence, was made available by the author [30] and it is this version that is used in the comparison with the data. The Pomeron-scattering option of ARIADNE was switched off.

\section{Correction Procedure}

The Monte Carlo samples described above were used to correct the differential $\phi$ distributions for detector effects. To minimise the sensitivity of the correction procedure to the underlying theoretical input of the Monte Carlo generator, the data were fitted using the following approach. Rather than correcting the data directly, a theoretical distribution $T\left(\phi_{T} ; \vec{a}\right)$ was used to represent the corrected azimuthal angle, $\phi_{T}$, distribution. The parametrisation $T\left(\phi_{T} ; \vec{a}\right)$ used in the analysis is that given in equation (11) plus an additional $D \sin \phi$ term, so that $\vec{a}$ here denotes the set of parameters $A, B, C$ and $D$. The set of parameters, $\vec{a}$, was determined by minimising the quantity

$$
\chi^{2}=\sum_{\phi_{M}} \frac{\left(P_{\mathrm{meas}}\left(\phi_{M}\right)-P_{\mathrm{rec}}\left(\phi_{M} ; \vec{a}\right)\right)^{2}}{\sigma_{M}^{2}},
$$

where $P_{\text {meas }}\left(\phi_{M}\right)$ is the measured $\phi$ distribution and $P_{\mathrm{rec}}\left(\phi_{M} ; \vec{a}\right)$ is the theoretical distribution smeared for detector effects. The term $\sigma_{M}^{2}$ is the sum in quadrature of the uncertainties of $P_{\text {meas }}$ and $P_{\text {rec }}$. The detector smearing takes account of: (i) migration between different $(x, y)$ bins, (ii) track reconstruction efficiency and the $\phi_{T}-\phi_{M}$ migration matrix and (iii) correction factors for 
pair conversion, $K^{0}$ decay etc. These smearing coefficients were obtained from generated and reconstructed Monte Carlo events.

For a bin size of $\pi / 5$ radians, the efficiency for a generated $\phi$ to be reconstructed in the same bin as generated is $\gtrsim 55 \%$. The purity of the reconstructed $\phi$ bin (i.e. the percentage of the tracks reconstructed in a bin coming from particles generated in the same bin) is $\gtrsim 60 \%$.

It should be noted that in this procedure a corrected experimental $\phi$ distribution is not determined. Instead the parameterisation of the true $T\left(\phi_{T} ; \vec{a}\right)$ distribution is fitted directly to the data. An important feature of this method is the fact that the $\phi_{T}-\phi_{M}$ migration matrix depends only weakly on the generated $\phi$ distribution, and is essentially determined by the experimental inefficiencies, which are well simulated. Because significant off-diagonal elements are present in the matrix, this fitting technique was used for the main analysis.

The more conventional bin-by-bin correction method was used as a check. Though this method is expected to be more dependent on the underlying physics in the generated distribution it has the advantage of producing a corrected experimental $\phi$ distribution. Correction factors, $F(\phi)$, were obtained from the Monte Carlo simulation by comparing the generated distributions before the detector and trigger simulation with the "observed" distributions after these simulations followed by the same reconstruction, selection and analysis as the real data. The correction factor for $\phi$ is defined as the ratio of the generated and observed differential $\phi$ distributions:

$$
F(\phi)=\left(\frac{1}{N} \frac{d n}{d \phi}\right)_{\mathrm{gen}} /\left(\frac{1}{N} \frac{d n}{d \phi}\right)_{\mathrm{obs}},
$$

where $N$ is the number of Monte Carlo events and $n$ the number of charged particles in the $(x, y)$ range. The correction factors are in the range 0.8-1.2.

\section{$5 \quad$ Systematic Checks}

The major systematic errors can be divided into three types: uncertainties due to event reconstruction and selection; to track selection; and to the modelling of the hadronic system. No single systematic uncertainty was larger than the statistical error in the mean of either $\cos \phi$ or $\cos 2 \phi$. For both mean values, the largest effects, which approached the statistical uncertainties, were associated with: the inclusion of tracks not associated with the primary vertex; the use of different correction procedures for the energy scattered into the calorimeter from the interaction of energetic particles in the forward direction; the use of an alternative approach to correct for energy losses due to inactive material between the calorimeter and the interaction vertex; and the $z_{h}$ cut being lowered by an amount commensurate with its reconstruction resolution. The mean value of $\cos 2 \phi$ was also reduced by its statistical uncertainty for $p_{c}>1.0 \mathrm{GeV}$ when LEPTO was used to correct the data. The upper and lower uncertainties extracted from these systematic checks were added separately in quadrature.

Three additional cross-checks were made on the measurement that were not included in the systematic uncertainties. The analysis was repeated using: (i) the bin-by-bin correction; (ii) the measured positron energy in place of that calculated from the DA method, to check the sensitivity to the boost, and (iii) only events without bremsstrahlung from the Monte Carlo sample. In each case the results were consistent, within statistical errors, with those from the original analysis. 


\section{Results}

Figure 2 shows the differential $\phi$ distributions of charged hadrons for four values of the $p_{T}$ cut, $p_{c}$, in the hadronic centre-of-mass. The results obtained from the main analysis method (full line) and the bin-by-bin correction method (points) are seen to agree. At low $p_{c}$, a clear $\cos \phi$ term is observed. As the value of $p_{c}$ is increased a $\cos 2 \phi$ term becomes evident.

Figure 3 shows the moments $\langle\cos \phi\rangle$ and $\langle\cos 2 \phi\rangle$ as a function of $p_{c}$. They were calculated from the fit using $\langle\cos \phi\rangle=B / 2 A$ and $\langle\cos 2 \phi\rangle=C / 2 A$. The $\sin \phi$ term is consistent with zero independent of the value chosen for $p_{c}$. The statistical errors are those obtained from fitting the data. The value of $\langle\cos \phi\rangle$ is negative and decreases in magnitude as $p_{c}$ is increased. In contrast, the value of $\langle\cos 2 \phi\rangle$ is positive and rises as $p_{c}$ is increased. Figure 3 also shows predictions of the Lund Monte Carlo programs (LEPTO and ARIADNE). The data have larger absolute values of $\langle\cos \phi\rangle$ than predicted by LEPTO, though for higher values of $p_{c}$ the agreement is reasonable. The dependence of $\langle\cos 2 \phi\rangle$ on $p_{c}$ is weaker in the LEPTO generator than in the data. Imposing a flat $\phi$ dependence on the matrix elements implemented in the LEPTO program, in order to investigate any asymmetry arising from purely QCD coherence effects, results in behaviour different from the data; both moments are almost zero for all values of $p_{c}$. The ARIADNE Monte Carlo predictions tend to have smaller absolute values of $\langle\cos \phi\rangle$ than the data, whilst the $\langle\cos 2 \phi\rangle$ term is in reasonable agreement with the data.

Figure 1 compares the data with two LO QCD calculations. Both calculations were made with $Q$ as the appropriate scale, with the Binnewies et al. LO fragmentation function [31] and with the CTEQ4 LO proton parton densities [24. The LO calculations result in a qualitatively similar behaviour to the LEPTO and ARIADNE Monte Carlo generator predictions.

The analytic calculation from ZEUS (based on the calculation of Chay et al. [5]) includes an estimation of the non-perturbative contribution, from intrinsic $k_{T}$ and hadronisation $p_{T}$, and integrates over the whole kinematic range. The results of Ahmed \& Gehrmann are purely perturbative at leading order in $\alpha_{s}$ and are evaluated at the mean values $\langle x\rangle=0.022$ and $\left\langle Q^{2}\right\rangle=750 \mathrm{GeV}^{2}$ of the data. The different implementations account for the observed difference in the two predictions; using $\langle x\rangle$ and $\left\langle Q^{2}\right\rangle$ in the ZEUS perturbative calculation leads to agreement with the Ahmed \& Gehrmann calculation.

There are perturbative and non-perturbative contributions to both $\langle\cos \phi\rangle$ and $\langle\cos 2 \phi\rangle$. For $\langle\cos \phi\rangle$, the non-perturbative contribution to the ZEUS calculation is at most $20 \%$ at low $p_{c}$ for a mean intrinsic $k_{T}$ of $0.5 \mathrm{GeV}$ and a nonperturbative fragmentation $p_{T}$ of $0.5 \mathrm{GeV}$. However, this fraction depends on the values of $k_{T}$ and $p_{T}$ used. For $\langle\cos 2 \phi\rangle$, this non-perturbative contribution is negligible, even for mean values of $k_{T}=0.9 \mathrm{GeV}$ and $p_{T}=0.9 \mathrm{GeV}$. It can be concluded that the observation of a significant $\langle\cos 2 \phi\rangle$ term is clear evidence for a perturbative contribution to the azimuthal asymmetry.

The sensitivity to higher-order corrections was examined at the partonic level using the DISENT Monte Carlo program [32]. This study suggests that higherorder corrections are large (factors of 2-3) at low $p_{c}$ for the $\langle\cos \phi\rangle$ term, resulting in larger negative values. For the $\langle\cos 2 \phi\rangle$ term they are much smaller. This may explain the disagreement between the data and the LO QCD models for $\langle\cos \phi\rangle$ at low $p_{c}$. 


\section{Conclusions}

The azimuthal asymmetries in the deep inelastic electroproduction of single particles have been measured at HERA in the hadronic centre-of-mass frame in the kinematic region, $\langle x\rangle=0.022$ and $\left\langle Q^{2}\right\rangle=750 \mathrm{GeV}^{2}$. For hadrons produced at large transverse momenta, the measured value for $\langle\cos \phi\rangle$ is negative and is in agreement with QCD predictions. The moment $\langle\cos 2 \phi\rangle$ has been measured here for the first time and is non-zero and positive. It increases as a function of the minimum particle transverse momentum, as expected from QCD. Since the non-perturbative contribution to $\langle\cos 2 \phi\rangle$ is predicted to be negligible, this measurement provides clear evidence for a perturbative QCD contribution to the azimuthal asymmetry.

\section{Acknowledgements}

The strong support and encouragement of the DESY Directorate have been invaluable, and we are much indebted to the HERA machine group for their inventiveness and diligent efforts. The design, construction and installation of the ZEUS detector have been made possible by the ingenuity and dedicated efforts of many people from inside DESY and from the home institutes who are not listed as authors. Their contributions are acknowledged with great appreciation. We would like to thank Thomas Gehrmann and Mike Seymour for useful discussions.

\section{References}

[1] H. Georgi and H. D. Politzer, Phys. Rev. Lett. 40 (1978) 3.

[2] R. N. Cahn, Phys. Lett. B78 (1978) 269.

[3] G. Köpp, R. Maciejko and P. M. Zerwas, Nucl. Phys. B144 (1978) 123.

[4] A. Mendez, Nucl. Phys. B145 (1978) 199.

[5] J. Chay et al., Phys. Rev. D45 (1992) 46, J. Chay et al., Phys. Lett. B269 (1991) 175 .

[6] K. A. Oganessyan et al., Eur. Phys. J. C5 (1998) 681.

[7] M. Ahmed and T. Gehrmann, Phys. Lett. B465 (1999) 297.

[8] EMC Collab., M. Arneodo et al., Z. Phys. C34 (1987) 277.

[9] E665 Collab., M. R. Adams et al., Phys. Rev. D48 (1993) 5057.

[10] ZEUS Collab., M. Derrick et al., Phys. Lett. B293 (1992) 465;

ZEUS Collab., M. Derrick et al., Z. Phys. C63 (1994) 391.

[11] ZEUS Collab., The ZEUS Detector, Status Report 1993, DESY 1993.

[12] N. Harnew et al., Nucl. Inst. Meth. A279 (1989) 290;

B. Foster et al., Nucl. Phys. B (Proc. Suppl.) 32 (1993) 181;

B. Foster et al., Nucl. Inst. Meth. A338 (1994) 254.

[13] M. Derrick et al., Nucl. Inst. Meth. A309 (1991) 77;

A. Andresen et al., Nucl. Inst. Meth. A309 (1991) 101;

A. Bernstein et al., Nucl. Inst. Meth. A336 (1993) 23. 
[14] F. Jacquet and A. Blondel, Proceedings of the study for an ep facility for Europe, DESY 79/48 (1979) 391.

[15] S. Bentvelsen, J. Engelen and P. Kooijman, Proceedings of the 1991 Workshop on Physics at HERA, DESY Vol. 1 (1992) 23;

K. C. Hoeger, ibid. 43.

[16] ZEUS Collab., J. Breitweg et al., Eur. Phys. J. C11 (1999) 447.

[17] ZEUS Collab., M. Derrick et al., Z. Phys. C72 (1996) 399.

[18] R. Brun et al., GEANT3, CERN DD/EE/84-1 (1987).

[19] K. Charchuła, G. Schuler and H. Spiesberger, Comp. Phys. Comm. 81 (1994) 381;

H. Spiesberger, DJAngo6 version 2.4 - A Monte Carlo Generator for Deep Inelastic Lepton Proton Scattering Including QED and QCD Radiative Effects, 1996, available on WWW:

http://www.desy.de/ hspiesb/django6.html

[20] A. Kwiatkowski, H. Spiesberger, and H.-J. Möhring, Comp. Phys. Commun. 69 (1992) 155;

H. Spiesberger, HERACLES - An Event Generator for ep Interactions at HERA Including Radiative Processes (Version 4.6), 1996, available on WWW:

http://www.desy.de/ hspiesb/heracles.html

[21] L. Lönnblad, Comp. Phys. Comm. 71 (1992) 15.

[22] B. Andersson et al., Phys. Rep. 97 (1983) 31.

[23] T. Sjöstrand, Comp. Phys. Comm. 82 (1994) 74;

T. Sjöstrand, CERN-TH 7112/93 (revised August 1995).

[24] H. L. Lai et al., Phys. Rev. D55 (1997) 1280.

[25] ZEUS Collab., M. Derrick et al., Z. Phys. C65 (1995) 379.

[26] H1 Collab., S. Aid et al., Nucl. Phys. B470 (1996) 3.

[27] G. Ingelman, A. Edin and J. Rathsman, Comp. Phys. Comm. 101 (1997) 108.

[28] L. Lönnblad, LU TP 99-22, hep-ph/9908368.

[29] A. Edin, G. Ingelman and J. Rathsman, Phys. Lett. B366 (1996) 371.

[30] L. Lönnblad, private communication.

[31] J. Binnewies, B.A. Kniehl and G. Kramer, Phys. Rev. D52 (1995) 4947.

[32] S. Catani and M. H. Seymour, Nucl. Phys. B485 (1997) 291, erratum-ibid B510 (1997) 503. 


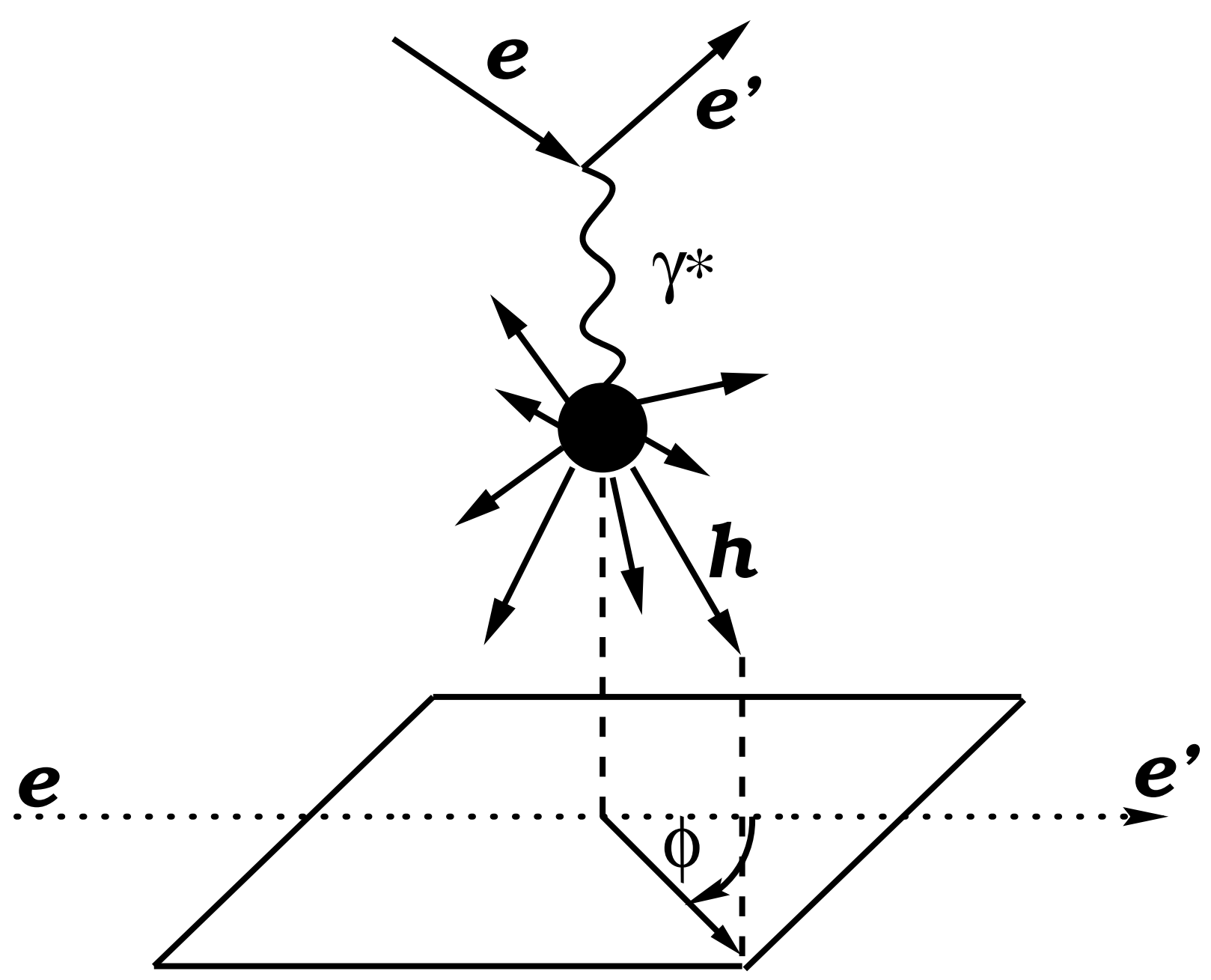

Figure 1: Definition of the azimuthal angle $\phi$. The incoming lepton is denoted by $e$, the scattered lepton by $e^{\prime}$, the exchanged boson by $\gamma^{*}$ and the outgoing hadrons or partons by $h$. The dotted line represents the intersection of the $e-e^{\prime}$ scattering plane with the transverse plane. 
ZEUS 1996-97

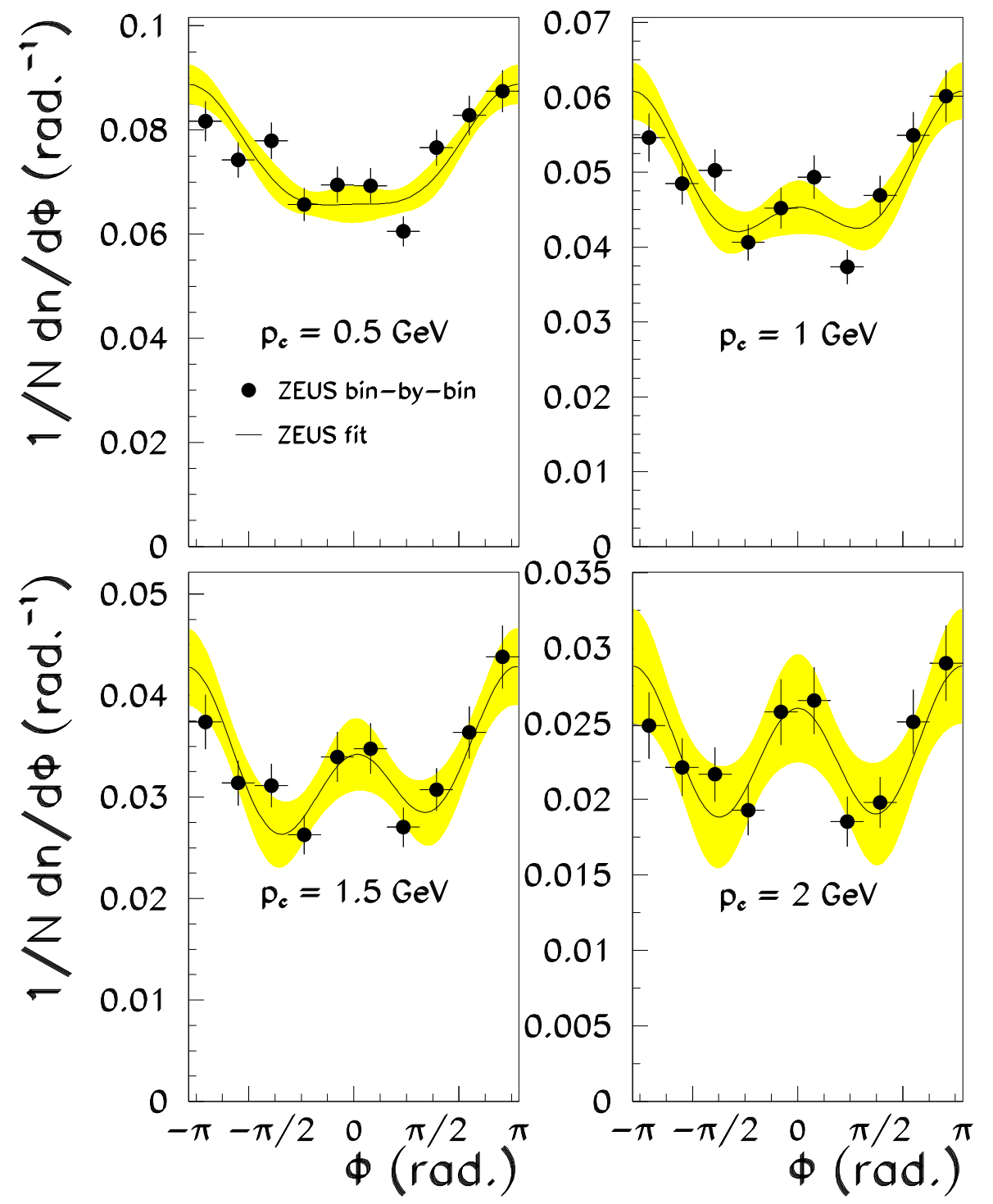

Figure 2: The differential $\phi$ distributions obtained for four values of the $p_{T}$ cut, $p_{c}$, in the hadronic centre-of-mass frame in the kinematic region $0.01<x<0.1$ and $0.2<y<0.8$ for charged hadrons with $0.2<z_{h}<1.0$. The full line (with accompanying statistical error band) is the result obtained from the main analysis using the unfolding technique. The data points were corrected using a bin-by-bin procedure (only statistical errors are shown). 

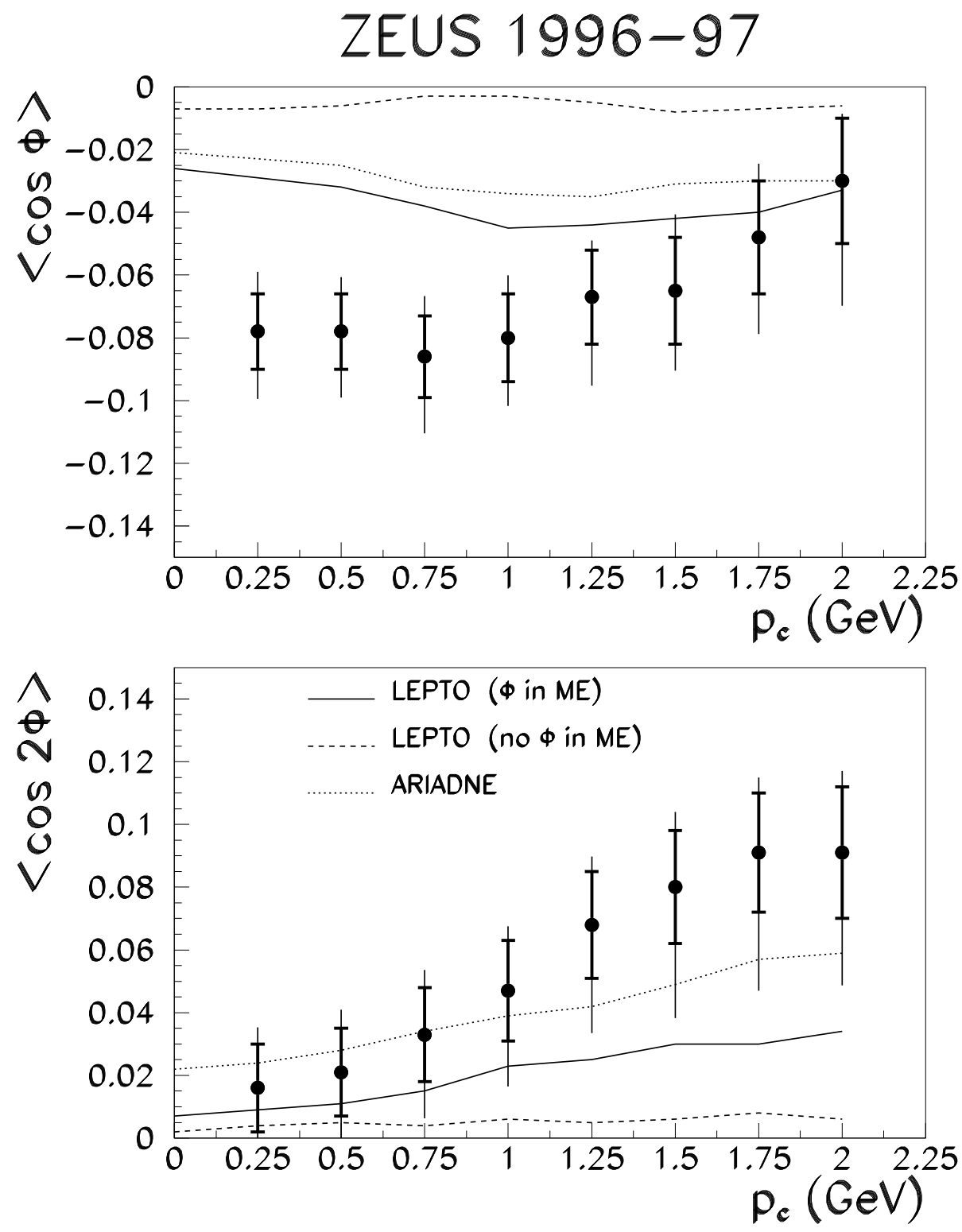

Figure 3: The values of $\langle\cos \phi\rangle$ and $\langle\cos 2 \phi\rangle$ are shown as a function of $p_{c}$ in the kinematic region $0.01<x<0.1$ and $0.2<y<0.8$ for charged hadrons with $0.2<z_{h}<1.0$. The inner error bars represent the statistical errors, the outer are statistical and systematic errors added in quadrature. The lines are the predictions from LEPTO (full line), LEPTO with flat $\phi$ dependence (dashed line) and ARIADNE (dotted line) Monte Carlo models. 
ZEUS 1996-97
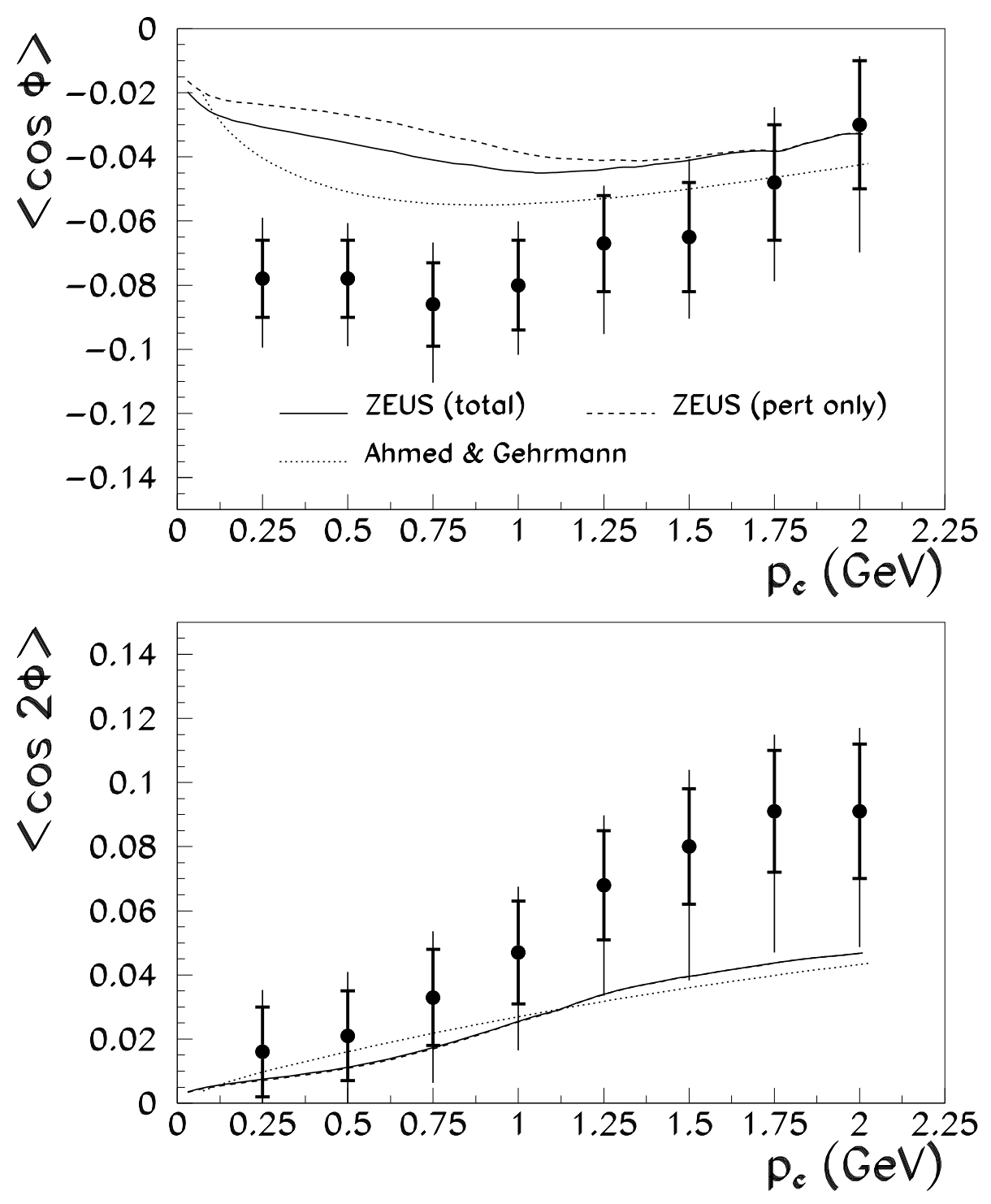

Figure 4: The values of $\langle\cos \phi\rangle$ and $\langle\cos 2 \phi\rangle$ are shown as a function of $p_{c}$ in the kinematic region $0.01<x<0.1$ and $0.2<y<0.8$ for charged hadrons with $0.2<z_{h}<1.0$. The inner error bars represent the statistical errors, the outer are statistical and systematic errors added in quadrature. The lines are the LO predictions from ZEUS with perturbative and non-perturbative contributions (full line), ZEUS with the perturbative contribution only (dashed line) and Ahmed \& Gehrmann (dotted line - see text for discussion). For the case of $\langle\cos 2 \phi\rangle$, the ZEUS total and perturbative predictions are almost identical. 